\title{
La formación médica en comunicación de malas noticias: Una revisión narrativa.
}

\section{The medical training in breaking bad news: A narrative review.}

\author{
Luis Alfonso Díaz-Martínez ${ }^{*}$, Mitzy Helein Cuesta Armesto², María José Díaz \\ Rojas $^{3}$
}

1 Profesor Titular, Escuela de Medicina, Facultad de Salud, Universidad Industrial de Santander, Bucaramanga; Estudiante, Maestría en Educación Médica, Facultad de Medicina, Universidad de la Sabana, Chía, Colombia; e-mail: ladimar@uis.edu.co. ORCID ID: 0000-0002-4498-6639.

2 Estudiante, Programa de Medicina, Escuela de Medicina, Universidad Industrial de Santander, Bucaramanga, Colombia. ORCID ID: 0000-0003-0930-2198.

3. Estudiante, Programa de Medicina, Escuela de Medicina, Universidad Industrial de Santander, Bucaramanga, Colombia. ORCID ID: 0000-0001-7283-4887.

* Correspondencia: Escuela de Medicina, Facultad de Salud, Universidad Industrial de Santander, Bucaramanga, Colombia. Carrera 32 No. 29-31, edificio administrativo, oficina 421. Correo electrónico: ladimar@uis.edu.co.

Recibido: 12/08/2020; Aceptado: 21/09/2020; Publicado: 23/09/2020

Resumen: Una parte fundamental de la atención en salud es la comunicación con los pacientes y sus familias, proceso crítico a la hora de dar malas noticias. Desafortunadamente, muchos médicos no reciben entrenamiento específico durante su pregrado, desarrollando, muchas veces en forma inadecuada, sus habilidades para hacerlo. Este artículo presenta las bases conceptuales, pedagógicas y didácticas para formular cursos o actividades formales de entrenamiento en dar malas noticias en un programa de Medicina. Se incluye una síntesis de lo que se conoce sobre la percepción que tienen médicos, estudiantes y pacientes sobre el dar o recibir malas noticias, sobre los protocolos existentes y sobre las experiencias documentadas sobre tal entrenamiento.

Palabras clave: Comunicación en Salud; Dar Malas Noticias; Divulgación de la Verdad; Relación Médico-Paciente; Educación Médica.

\begin{abstract}
In health care, communication with patients and their families is essential, and a critical process when breaking bad news. Unfortunately, many doctors do not receive specific training during their undergraduate studies, often developing their skills in an empirical way to do so. This article presents the conceptual, pedagogical, and didactic bases for formulating courses or training activities to break bad news in every medicine program. It includes a synthesis of what is known within physicians, students and patients perception about this task, on existing protocols and on documented experiences of such training.
\end{abstract}

Keywords: Health Communication; Break Bad News; Truth Disclosure; ProfessionalPatient Relations; Medical Education

\section{Introducción}

Se considera que un médico es competente cuando hace uso habitual y juicioso de la comunicación, el conocimiento, las habilidades técnicas, el razonamiento clínico, las emociones, los valores y la reflexión en la práctica diaria en beneficio de la persona y 
la comunidad a la que se sirve (1). Así, la comunicación es pilar importante de esta encomienda, para la cual hay que propiciar en los médicos ciertas habilidades que les permita hacerlo de forma exitosa y eficiente, habilidades que son específicas, integrativas, duraderas y focalizadas en el desempeño; por supuesto, pueden aprenderse y son mensurables (2).

La comunicación con los pacientes y sus familias, dada la carga emocional que contiene, es más compleja de lo que parece (3). Durante la formación de los profesionales de la salud se obvia muchas veces este apartado, dejando el desarrollo de las competencias en comunicación al azar, de tal forma que, ora como estudiante, ora como profesional, el médico se ha de enfrentar a dar malas noticias de manera instintiva, lo que puede generar errores y problemas en la forma como se abordan los pacientes (4). La evidencia muestra que los pacientes con mucha frecuencia están insatisfechos con la forma como los profesionales les advierten de su condición clínica y con la cantidad de información que se les suministra, lo que lleva a inconformidad, menor credibilidad, y, en algunos casos extremos, a querellas legales (5), en la medida que la perspectiva y habilidades que los médicos desarrollan, ya sea empírica o guiada por el currículo, no está centrada en el paciente sino tan solo desde su perspectiva y necesidades, por no decir, desde sus miedos e incompetencias (6-8).

Existe la obligación ética para que el entrenamiento en dar malas noticias sea abordado desde lo curricular de manera explícita y con calidad $(9,10)$, lo que hace necesario que se concreten propuestas pedagógicas culturalmente sensibles en programas médicos de pre y postgrado (11-15). Idealmente, estas propuestas han de permitirle al estudiante afrontar su entrenamiento con actividades académicas eficientes que les permitan evaluar el cambio que tienen en las destrezas, actitudes y sentimientos que experimentan al comunicar malas noticias, en una acción que debe estar centrada en el receptor de la información adversa (16-18). Este artículo contiene una serie de planteamientos que buscan, desde el reconocimiento de las diferentes perspectivas de los actores implicados, dar elementos de juicio a la hora de formular un programa de entrenamiento en dar malas noticias en una escuela o facultad médica, incluyendo ejemplos significativos de las experiencias documentadas sobre el entrenamiento en este tema, especialmente de estudiantes del grado en Medicina.

\section{Percepciones de los actores al dar o recibir malas noticias}

Se define a las malas noticias como cualquier información que potencialmente puede producir sentimientos de desamparo, representar una amenaza para la salud mental o física de un individuo o puede afectar en forma negativa su visión u opciones de su propio futuro (5). Esto es subjetivo y no depende del juicio de quien da la noticia, sino de la percepción del receptor de la información sobre la gravedad de la condición (19). El ejercicio de la comunicación en el contexto de dar malas noticias es claramente comunitario, de tal forma que el aprendizaje en este escenario tiene lugar a través de la interacción social en entornos culturales específicos $(14,20)$. Interactuar con los pacientes y reflexionar con los demás miembros de la comunidad de práctica hace que el aprendizaje sea significativo en la medida que el estudiante se involucra en la solución de problemas o la realización de tareas, haciendo que desarrolle un pensamiento crítico al respecto, de lo que no se escapa el hecho de dar o ser testigo de dar malas noticias (21).

\subsection{La perspectiva de los médicos graduados}

En principio, se consideraba que dar información clínica desfavorable causaría daño en el paciente: Hipócrates y Platón concordaban en que una "revelación 
honesta" podría tener como resultado el empeoramiento del pronóstico del paciente (22). Esta idea persistió hasta el siglo XIX; en 1847, la Asociación Médica Estadounidense planteaba que algunas acciones médicas podrían acortar la vida de un paciente, incluyendo las palabras que empleaba y cómo las utilizaba (23). Aunque desde los años 20 del siglo XX se comenzó a plantear el problema de comunicar o no a los pacientes su diagnóstico en el contexto de cáncer, no fue sino hasta los años 60 que se empezó a buscar evidencia sobre si los pacientes debían conocer la verdad sobre su diagnóstico, o sobre la mejor manera para hacerlo; Oken, en 1961, reveló que el $88 \%$ de los médicos omitían decirles a sus pacientes el diagnóstico de cáncer de manera rutinaria, con la creencia de que así se disminuía la eventualidad del suicidio y se lograba mayor adherencia al tratamiento (24). Otra práctica usual era informar a los cuidadores sobre la condición del paciente y dejar a su criterio la decisión de si compartir esto o no con el afectado (25); ya en el siglo XXI, en Polonia hacia 2013, el $28 \%$ de los médicos y el $32 \%$ de los estudiantes pensaban lo mismo (26).

A pesar de conocer las necesidades de los pacientes, la tarea de satisfacerlas sigue siendo difícil puesto que para el profesional también significa un esfuerzo emocional. Dentro de las mayores dificultades que enfrenta se incluyen el miedo a ser culpado, a enfrentarse a una reacción que no ha anticipado, a expresar emociones inapropiadas, a que la noticia desequilibre al receptor, a no ser capaz de responder todas las preguntas, a desolar al paciente acabando con su optimismo, o a la vergüenza por haber sido positivo anteriormente (27). Por otro lado, la actitud del médico al dar malas noticias puede verse afectada por entrenamiento deficiente o experiencias adversas, también por sus convicciones culturales, filosóficas y religiosas sobre la muerte y la adversidad (28); además, se espera que el médico tenga control de sus propias emociones, que evite la reactividad ante la respuesta del paciente, que use de forma adecuada los silencios y la expresión no verbal, buscando generar un buen acto de comunicación que fluya en ambas direcciones (29).

De forma paradójica, en estudios hechos en distintos lugares del mundo, la cantidad de profesionales con entrenamiento ex profeso sobre dar malas noticias es escaso: aún con grupos de profesionales que se enfrentan continuamente a este acto, menos del $40 \%$ reconocen haber sido entrenados de manera formal; por ejemplo, entre los oncólogos, la proporción de médicos entrenados oscila entre 6 y 24\%, lo que equivale a aceptar como relevante la afirmación de la mayoría de los clínicos, quienes reconocieron que adelantaban de forma instintiva esta práctica por la falta de entrenamiento durante su formación (30-32).

La situación en Latinoamérica es igualmente desconcertante. En un estudio realizado en Cuba, se encontró que cerca del $60 \%$ de los médicos de atención primaria reconocen la importancia de la información verbal pero sin tener en cuenta el impacto que tiene la comunicación no verbal; además, casi el 10\% menciona que su estrategia de aprendizaje se basó en la "intuición" y el "autoestudio" (33). De hecho, el fenómeno de la baja proporción de capacitación específica se ha documentado en la región (3437).

\subsection{Las propuestas y necesidades de los pacientes y sus allegados}

A pesar del reto que implica para los profesionales de la salud el dar malas noticias, no es el médico el principal actor en el proceso, sino el receptor de la información (24). La forma en la que son dadas las malas noticias determina la comprensión de la información por parte del paciente, su satisfacción con el cuidado médico, la adherencia al tratamiento, su nivel de esperanza y, con esto, su adaptación 
psicológica a la situación, haciendo que aumente o disminuya su vulnerabilidad a la ansiedad y la depresión (6). A ello está asociada la situación incómoda que supone el encuentro frontal con tópicos muy sensibles para los seres humanos, como el límite de la vida o el sufrir una incapacidad, que generan frustración (38-41), y las reacciones psicológicas negativas propias de un proceso del duelo: negación, ira, negociación, depresión-resignación y aceptación (42). Por otro lado, la comunicación asertiva en el contexto de la interacción médico-paciente no sólo mejora la percepción de satisfacción de los dolientes con la atención, sino que ayuda al profesional a identificar situaciones de riesgo de manera temprana y los hace más receptivos al tratamiento y a modificar las conductas perjudiciales (43). Esta comunicación debe tener en cuenta también lo que no se dice, prestando especial atención en tener contacto visual en intervalos razonables e interpretar de forma correcta sus reacciones (44).

Es clara la tendencia en los pacientes a preferir recibir la información sobre su diagnóstico de la forma más clara y completa posible: ya en 1982, el 96\% de norteamericanos preferían conocer la verdad sobre un posible diagnóstico de cáncer, y el $86 \%$ deseaba recibir, en caso de un pronóstico grave, una estimación realista sobre su pronóstico (13). Esto se repite en otros países, incluyendo regiones de cultura distinta a la occidental $(45,46)$. Es por ello que es importante el carácter terapéutico que tiene el conocimiento y el control que el paciente pueda ejercer sobre su propia enfermedad; esto alivia su sufrimiento, le da herramientas para involucrarse y tomar decisiones sobre su propio cuidado, sin olvidar que asegura la adherencia al tratamiento (47), aunque los pacientes con baja escolaridad, edad avanzada, varones y con las condiciones más severas tienden a escoger un rol pasivo (16).

Benítez y Asencio afirman que en procesos esperables que determinan la negación, se dispone la forma como el médico comunica la verdad, pues los pacientes huyen de la realidad como mecanismo de defensa $\mathrm{y}$, en este momento, el enfrentamiento con la realidad genera mayor ansiedad (48); esto es algo definido como "la verdad soportable", un punto más allá de la cual el paciente entra en negación y la defiende a ultranza (49). De allí que se propongan ciertas etapas en el proceso de información como una guía para acompañar la situación de manera abierta, teniendo especial cuidado en las reacciones del paciente para avanzar de manera dirigida según como se desenvuelva la entrevista (48). Independiente de lo anterior, en el momento de recibir la noticia, los aspectos que más valoran los pacientes son que se les dé la información en persona, que el médico les preste plena atención, incluyendo contacto visual, que se les explique las mejores opciones de tratamiento, que se tenga una actitud proactiva hacia buscar las mejores alternativas para su condición y que se esté dispuesto a contestar las preguntas que surjan (50).

En la población infantil, el asunto es más complejo en la medida que las malas noticias se comparten más con padres y familiares que con los pacientes mismos. Quizás la mejor ilustración del fenómeno es la que plantean 735 padres al cuidado de niños con enfermedades crónicas, quienes describieron e hicieron recomendaciones a los médicos acerca de lo que estos pueden esperar de aquellos durante la atención de sus hijos. Plantean que durante la atención de sus hijos se presenta una situación irónica, por no decir doblemente contradictoria, pues a pesar de ser considerados ignorantes ante la enfermedad de sus hijos se espera de ellos cumplir con la responsabilidad primaria de coordinar su cuidado. También mencionan que, aunque el médico puede conocer los aspectos teóricos de la enfermedad de sus hijos, son ellos quienes viven la clínica, por lo que conocen mejor la enfermedad y han aprendido estrategias de adaptación; ello implica, desde su perspectiva, que el médico no 
debería subestimarlos y, sobre todo, no considerarlos un obstáculo en circunstancias difíciles, aún si están en situación de gran demanda emocional. Aluden a la necesidad de involucrar al niño utilizando términos apropiados para la edad, para que conozca y entienda mejor su propia enfermedad, así como respetar y no juzgar las decisiones de los padres. Según su percepción, el médico puede facilitar el trabajo del cuidador desde la integralidad en la atención; lo dicen con claridad: "vean a cada niño como una persona completa" (51).

\subsection{Lo que perciben los estudiantes de su entrenamiento en dar malas noticias.}

En 1995 se creó la primera guía sobre cómo anunciar malas noticias, pero menos del $2 \%$ de los artículos que se han publicado sobre el tema se enfocan en crear un sistema que las integre en la academia (52). Diez años después, dos terceras partes de los estudiantes habían sido testigos de cómo un médico informa malas noticias, pero menos del 15\% habían recibido algún tipo de entrenamiento formal en la entrega de malas noticias (53). Con todo, los médicos y estudiantes que reciben formación sobre dar malas noticias muestran progreso en el campo hasta cinco años después del entrenamiento, pero se mantienen distantes y reservados a explorar los problemas psicosociales del paciente que influyen en la forma como estos reciben la noticia (54).

En la población estudiantil hay ambivalencia respecto a tomar cursos sobre dar malas noticias. Algunas posturas apuntan a que la formación integral del médico requiere el aprendizaje de estas habilidades, mientras que otras muestran que los cursos disponibles no son considerados "ciencia pura", que el tiempo de entrenamiento es insuficiente o que tales habilidades se adquieren con facilidad por "sentido común" (55). Por otro lado, es más frecuente encontrar receptivos a los estudiantes de primer año en comparación con los de años avanzados (56), en donde la principal preocupación de los estudiantes más avanzados es sobre la metodología a dominar, mientras que los más jóvenes temen más a las repercusiones emocionales del paciente y sus familiares al discutir sobre enfermedades terminales (6).

Para desarrollar correctamente esta disciplina se necesita cultivar la empatía desde los primeros momentos que el estudiante entra en contacto con los pacientes y sus familias (57), entendiendo ésta como la capacidad de comprender las emociones de otros individuos y saber reflejar esa comprensión (58). La necesidad de que distingan la línea que separa la empatía de la distancia profesional crea tensiones en los estudiantes de la salud en el momento de referirse a los pacientes en circunstancias que los comprometan emocionalmente (41). Ahora bien, se puede desarrollar la empatía desde cuatro habilidades importantes: discriminar el impacto que tendrá la noticia para el paciente, ser capaz de mitigar el daño percibido por el enfermo, modificar el contenido del mensaje de manera que no resulte ofensivo, y tener la habilidad lingüística para comunicarlo (59).

Sin embargo, a pesar del reconocimiento de la importancia de la empatía en la educación de un estudiante de medicina, ésta en realidad disminuye significativamente durante la carrera hasta la graduación (60). De allí emerge una paradoja: mientras se reconoce y estimulan los valores relativos a empatía, compasión y altruismo, junto con un compromiso tácito con una ética de desapego, desinterés propio y objetividad, "aún los médicos experimentados a veces carecen de la capacidad para reconocer las situaciones difíciles de sus pacientes, para extender la empatía hacia aquellos que sufren y para unirse con los pacientes en su enfermedad de manera honesta y valiente" (61). 
Por otro lado, los estudiantes reconocen que las herramientas no verbales son fácilmente modificables para ajustarse a su propio estilo de comunicación; por ejemplo, que sentarse a la altura del paciente y mantener contacto visual brinda la percepción de que se le dedicará más tiempo a la entrevista y mejora la actitud del paciente y familiares. Aunque muchos aseguraron que la forma de comunicar es inherentemente buena para cada persona, coinciden en que el foco asertivo está no en lo que se dice, sino en cómo se dice. Los comentarios negativos se encontraban orientados a que la empatía no podía enseñarse, y que no era necesario para un buen comunicador recibir entrenamiento; además, la mayoría prefería el aprendizaje con el propio paciente que hacerlo apoyado en protocolos o la simulación (62).

\section{Enseñar a dar malas noticias}

Bain plantea que lo que hace grande a los profesores no es tanto lo que hacen sino lo que comprenden, pero que, ante todo, lo que creen que sus estudiantes pueden aprender (63). Las estrategias de aprendizaje sobre dar malas noticias incluyen desde cursos virtuales (64) hasta entrenamiento in vivo con pacientes y estudiantes de otras áreas de la salud (39). En todo caso, los estudiantes se preocupan por la pertinencia real de los resultados académicos que obtienen, especialmente cuando lo que usualmente toman son asignaturas electivas que demandan horarios y estrategias de aprendizaje distintos a los que encuentran con los métodos de enseñanza clínica presencial (65).

Los estudiantes con frecuencia sobreestiman sus capacidades antes de iniciar los cursos de formación (66); por ejemplo, un programa de entrenamiento en un centro médico de Holanda mostró que los alumnos pasaban de considerarse inconscientemente incompetentes a hacerse conscientes de su incompetencia, adjudicando su percepción adversa a la falta de apoyo emocional y realimentación durante la formación específica en dar malas noticias y el entrenamiento médico en general (64). A esto, la recomendación es entrenar los aprendices en cuatro puntos clave, que son retos fundamentales al dar malas noticias: conocer la condición clínica del paciente; ser capaz de proporcionar la información de tal forma que el paciente la pueda entender efectivamente; dar apoyo emocional al paciente y su familia; y a desarrollar una estrategia posterior que permita el logro de los objetivos que se esperan (39).

Existen al menos 14 protocolos para dar malas noticias (27) que en general orientan a tener en cuenta varios puntos: la preparación emocional del profesional y del ambiente; el conocer cuánto sabe y qué quiere saber el paciente; ser capaz de comunicarse correctamente, donde se desaconseja el uso de eufemismos; el comprender, estimular y valorar las reacciones y emociones del paciente; reconocer la necesidad de atención de morbilidad psicológica; el afrontar situaciones como la negación, las diferencias culturales o los familiares que presionan para mantener el silencio hacia su familiar enfermo; a ser culturalmente sensibles, y, finalmente, al facilitar la enseñabilidad de los modelos de suministrar malas noticias (11,67-69). Así, el proceso de aprendizaje en dar malas noticias requiere invertir tiempo para que se revise en forma detallada los encuentros experimentados al dar malas noticias, reales o no, de tal forma que sea posible comparar tal experiencia con los protocolos propuestos, lo que permitiría a los estudiantes hacer una reflexión deliberada que les ayude a entender mejor la dinámica del proceso y apropiarse del conocimiento y las habilidades, incluso si esta manera de aprendizaje llegase a contradecir la enseñanza formal (70). 
Cuando se piensa incluir entrenamiento en dar malas noticias en el currículo se debe tener en cuenta la brecha que existe entre la práctica médica y la experiencia simulada; los estudiantes reconocen las diferencias entre ambos ambientes y sus capacidades se ven limitadas si el curso solo utiliza el juego de roles como herramienta de aprendizaje (71). Una alternativa es el uso de la escritura reflexiva o la observación de pacientes como herramienta para el aprendizaje de actitudes empáticas, la cual ha mostrado que expresar sus emociones en papel y en privacidad sobre diferentes acontecimientos durante las rotaciones de tal forma que sea posible plasmar su posición frente a la muerte, el dolor y el sufrimiento, tiene un impacto positivo en el desarrollo de la empatía (69). De esto puede surgir la propuesta de implementar una discusión guiada con un facilitador que tenga mayor experiencia, capaz de aconsejar sobre estrategias para lidiar con estas situaciones, que incluya el análisis de los relatos contrastados con el estudio de los protocolos (72).

Ejemplos exitosos de este abordaje son uno que se desarrolló en Chile durante cinco años para estudiantes de quinto y sexto semestre de medicina en un taller con base en tres ejes: el manejo de situaciones difíciles, la comunicación de las malas noticias a pacientes y familiares, y el autocuidado del profesional. Al final del curso, el $76 \%$ de los asistentes se sintió muy satisfecho o satisfecho y ninguno reportó insatisfacción. Sin embargo, dentro de las críticas de los estudiantes estaba la necesidad no satisfecha de construir un esquema mental concreto del asunto y de querer tener más ejercicios, incluyendo juegos de roles y simulación (73). Otro fue el de un hospital de cáncer de Beijing en donde se realizó un programa de entrenamiento en habilidades comunicativas, actividad de gran aceptación entre los participantes, quienes mejoraron en forma significativa en muchas áreas, en especial en aquellas que les representaba algún tipo de conflicto, como hablar con el paciente de su muerte o darle soporte emocional (74). Finalmente, Servotte et al en Bélgica informaron, en un estudio con las características de un ensayo clínico controlado, que un curso de cuatro horas en el que se simulaba dar malas noticias, insertado en la rotación urgencias, hizo que los asistentes mejoraran sustancialmente en cuanto a autoeficacia, en el proceso de dar malas noticias y en la comunicación en general, frente a sus pares que no asistieron al curso (75). Para más ejemplo, incluyendo de uso de la virtualidad en el entrenamiento, puede consultarse las experiencias recientes de Guilera (76) o Flores-Funes en España (77), de Villela en Brasil (78), de Vermylen en los Estados Unidos (79), de Carrard (80) o de Schmitz en Suiza (81), o Papadakos en Grecia (82).

Uno de los problemas es el de evaluar el desenlace esperado, como puede ser la mejora en la empatía (83). Maguire plantea que durante los cursos sobre dar malas noticias es importante mostrar la evidencia de las deficiencias en la comunicación de manera consecuente, ofrecer evidencia sobre la efectividad en la adquisición de habilidades, y demostrar durante los talleres la evolución de los mismos participantes, promoviendo también la oportunidad de realizar simulaciones y brindar realimentación para reflejar los comportamientos de cada uno de los participantes (44). En la Universidad de Michigan, 21 estudiantes de medicina recibieron entrenamiento de manera estandarizada en dos escenarios diferentes; su desempeño fue comparado con el de 17 estudiantes sin experiencia de formación. Al final todos se enfrentaron a una paciente que simulaba la pérdida de su embarazo, quien al mismo tiempo era su evaluador. El estudio arrojó que los estudiantes entrenados tuvieron un mejor desempeño que el grupo control, sin que existan diferencias en los resultados obtenidos por los estudiantes entrenados con uno y otro escenario, lo que mostró que lo aprendido en una simulación es reproducible en otro tipo de situación (84). 
Un reto adicional en el entrenamiento de los médicos, incluyendo dar malas noticias, está dado por la organización de los servicios de salud que obliga a restringir el tiempo dedicado a la comunicación de malas noticias al paciente, que hace que se deba simplificar el encuentro, a que no se adelante en las condiciones ideales de privacidad, y que imposibilita el tiempo de catarsis que el médico necesita, lo que a largo plazo termina afectando su capacidad empática (85).

A manera de resumen sobre lo que ha de adelantarse con los estudiantes de medicina, es que este inicie un proceso de reflexión sobre el dar malas noticias a partir de una experiencia, seguido por un proceso de interpretación que se consolida al momento en que el estudiante le otorgue un sentido, explorando también los encuentros pasados, comparándolos con lo que hasta ese momento ha vivido (86). Es allí donde la realimentación que puedan recibir los estudiantes es clave para fortalecer el proceso que están viviendo (87). Este método permite a los estudiantes generar perspectivas propias y decidir de manera racional sobre los comportamientos que le parezcan adecuados y los que prefieren descartar, prestando además herramientas para construir una manera personal de dar malas noticias, que sea, por supuesto, positiva y eficiente, incluyendo un despliegue emocional completamente consciente (88).

Financiación: Este estudio no ha sido financiado por fuente externa a los recursos propios de los autores.

Declaración de conflicto de interés: Los autores declaran no tener ningún conflicto de intereses.

\section{Referencias}

1. Epstein RM, Hundert EM. Defining and assessing profesional competence. JAMA. 2002; 287(2):226-35. http://www.bristol-inquiry.org.uk/final report/report/sec2chap25 4.htm.

2. Rider EA, Keefer $\mathrm{CH}$. Communication skills competencies: Definitions and a teaching toolbox. Med Educ. 2006; 40(7):624-9. DOI: https://doi.org/10.1111/j.13652929.2006.02500.x.

3. Perron NJ, Sommer J, Louis-Simonet M, Nendaz M. Teaching communication skills: Beyond wishful thinking. Swiss Med Wkly. 2015; 145:w14064. DOI: https://doi.org/10.4414/smw.2015.14064.

4. Orgel E, McCarter R, Jacobs S. A failing medical educational model: A self-assessment by physicians at all levels of training of ability and comfort to deliver bad news. J Palliat Med. 2010; 13(6):677-83. DOI: https://doi.org/10.1089/jpm.2009.0338.

5. Williams-Reade J, Lobo E, Whittemore AA, Parra L, Baerg J. Enhancing residents' compassionate communication to family members: A family systems breaking bad news simulation. Fam Syst Heal. 2018; 36(4):203-27. DOI: https://doi.org/10.1037/fsh0000331.

6. Sykes N. Medical students' fears about breaking bad news. Lancet. 1989; 334(8662):564. DOI: https://doi.org/ 10.1016/S0140-6736(89)90688-0.

7. Brouwers $\mathrm{M}$, van Weel C, Laan R, van Weel-BAumgarten E. Training undergraduates skills in breaking bad news: How students value educators' feedback. J Cancer Educ. 2019; 34(6):1103-6. DOI: https://doi.org/ 10.1007/s13187-018-1415-8.

8. O'Neill BJ, Wyness MA. Learning about intreprofessional education: Student voices. J Interprof Care. 2004; 18(2):198-200. DOI: https://doi.org/10.1080/13561820410001686945.

9. Mohandas KM. Ethical dilemmas in breaking bad news. Med Ethics. 1995; 3(4):59-60. http://www.ncbi.nlm.nih.gov/entrez/query.fcgi? $\mathrm{cmd}=$ Retrieve\&db=PubMed\&dopt $=$ Citation\&list uids $=15586947$.

10. Williams-Read J, Lobo E, Whittemode AA, Baerg J, Mohandas KM, Afghani B, et al. Breaking bad news. Patient Educ Couns. 2016;9(1):1-9. DOI: https://doi.org/10.4103/jfmpc.jfmpc. 
11. Keefe-Cooperman K, Savitsky D, Koshel W, Bhat V, Cooperman J. The PEWTER Study: Breaking bad news communication skills training for counseling programs. Int J Adv Couns. 2018; 40(1):72-87. DOI: https://doi.org/10.1007/s10447-017-9313-z.

12. Ayers NE, Vydelingum V, Arber A. An ethnography of managing emotions when talking about life-threatening illness. Int Nurs Rev. 2017; 64(4):486-93. DOI: https://doi.org/10.1111/inr.12356.

13. Chittem M, Butow P. Responding to family requests for nondisclosure: The impact of oncologists' cultural background. J Cancer Res Ther. 2015; 11(1):174. DOI: https://doi.org/10.4103/0973-1482.140836.

14. Tan Kiak Min M. Beyond a Western bioethics in Asia and its implication on autonomy. New Bioeth. 2017; 23(2):154-64. DOI: https://doi.org/10.1080/20502877.2017.1345091.

15. Cushing AM. Learning patient-centred communication: The journey and the territory. Patient Educ Couns 2015; 98(10):1236-42. DOI: https://doi.org/10.1016/j.pec.2015.07.024.

16. Bishop TW, Gorniewicz J, Floyd M, Tudiver F, Odom A, Zoppi K. Innovative patient-centered skills training addressing challenging issues in cancer communications: Using patient's stories that teach. Int J Psychiatry Med. 2016; 51(4):357-66. DOI: https://doi.org/10.1177/0091217416659272.

17. Sanson-Fisher R, Hobden B, Carey M, Mackenzie L, Hyde L, Shepherd J. Interactional skills training in undergraduate medical education: ten principles for guiding future research. BMC Med Educ. 2019; 19:144. DOI: https://doi.org/10.1186/s12909-019-1566-2.

18. Lau-Koch C, Badch-Rosa A, Cldas-Bedin S. Malas noticias: Significados atribuidos en la práctica asistencial neonatal / pediátrica. Rev Bioética. 2017; 25(3):577-84. DOI: https://doi.org/10.1590/1983-80422017253214.

19. Halpern J. Empathy and patient-physician conflicts. J Gen Intern Med. 2007; 22(5):696-700. DOI: https://doi.org/10.1007/s11606-006-0102-3.

20. Ali AA. Communication skills training of undergraduates. J Coll Physicians Surg Pakistan. 2013; 23(1):10-5. DOI: https://doi.org/01.2013/JCPSP.1015.

21. Bleakley A. Broadening conceptions of learning in medical education: The message from teamworking. Med Educ. 2006; 40(2):150-7. DOI: https://doi.org/10.1111/j.13652929.2005.02371.x.

22. Rudnick A, Ezra Y, Melamed E. Breaking bad news and personality assessment. Patient Educ Couns. 2000; 41(2):157-60. DOI: https://doi.org/10.1016/S0738-3991(99)00106-8.

23. Ehsani M, Taleghani F, Hematti S, Abazari P. Perceptions of patients, families, physicians and nurses regarding challenges in cancer disclosure: A descriptive qualitative study. Eur J Oncol Nurs. 2016; 25:55-61. DOI: https://doi.org/10.1016/j.ejon.2016.09.003.

24. Oken D. What to tell cancer patients. JAMA. 1961; 175(13):1120-8. DOI: https://doi.org/10.1016/j.jacc.2017.03.299.

25. Kurer M, Zekri J. Breaking bad news: Can we get it right? Libyan J Med. 2008; 3(4):200-3. DOI: https://doi.org/ 10.4176/080825.

26. Leppert W, Majkowicz M, Forycka M. Attitudes of polish physicians and medical students toward breaking bad news, euthanasia and morphine administration in cancer patients. J Cancer Educ. 2013; 28(4):603-10. DOI: https://doi.org/10.1007/s13187-013-0553-2.

27. Ahmady AE, Sabounchi SS. A Suitable Model for Breaking Bad News: Review of recommendations. JMED Res. 2014; 2014:1-15. DOI: https://doi.org/10.5171/2014.776618.

28. Mostafazadeh-Bora M, Zarghami A. Breaking and sharing bad news in end of life: The religious and culture matters. J Relig Health. 2017; 56(5):1655-7. DOI: https://doi.org/10.1007/s10943-016-0249-0.

29. Borrel i Carrió F, Prados Castillejo JA. Comunicar malas noticias. Estrategias sencillas para situaciones complejas. Barcelona: Doyma; 1995.

30. Mostafavian Z, Shaye Z. Evaluation of physicians' skills in breaking bad news to cancer patients. J Fam Med Prim Care. 2018; 7:601-5. DOI: https://doi.org/4103/jfmpc.jfmpc 2518. 
31. Beckman HB, Markakis KM, Suchman AL, Frankel R. The doctor-patient relationship and malpractice. Lessons from plaintiff depositions. Arch Intern Med. 1994; 154(12):1365-70. Recuperado de https://jamanetwork.com/journals/jamainternalmedicine/article-abstract/ 618939.

32. Yip S, Meyers DE, Sisler JJ, Wycliffe-Jones K, Kucharski E, Elser C, et al. Oncology education for family medicine residents: a national needs assessment survey. BMC Med Educ. 2020; 20:283. DOI: https://doi.org/10.1200/jco.2018.36.15 suppl.11014.

33. Martín Hernández I, Trujillo Matienzo C. Comunicación de malas noticias a pacientes con enfermedades neurodegenerativas: habilidades del personal médico. Rev Latinoam Bioética. 2009; 9(2): 76-85. DOI: https://doi.org/10.18359/rlbi.1061.

34. Bardales AF, Querevalú DL, Maquera-Afaray J. Comunicación de malas noticias: Experiencia en un hospital del Perú. Rev Peru Med Exp Salud Púb. 2014; 31(3):601-10. Recuperado de http://www.scielo.org.pe/scielo.php?script=sci arttext\&pid=S1726-46342014000300030.

35. Payán EC, Montoya DA, Vargas JJ, Vélez MC, Castaño A, Krikorian A. Barriers and facilitating communication skills for breaking bad news: From the specialists' practice perspective. Colomb Med. 2009; 40(2):158-66. Recuperado de http://www.scielo.org.co/scielo.php?script=sci arttext\&pid=S1657-95342009000200003.

36. Rebello C, Marchetti MA, Lemonica L, Moreira GA. The P-A-C-I-E-N-T-E Protocol: An instrument for breaking bad news adapted to the Brazilian medical reality. Rev Assoc Méd Bras. 2017; 63(1):43-9. DOI: https://doi.org/10.1590/1806-9282.63.01.43

37. Herrera A, Ríos M, Manríquez JM, Rojas G. Entrega de malas noticias en la práctica clínica. Rev Med Chil. 2014; 142:1306-15. Recuperado de https://scielo.conicyt.cl/scielo.php? script=sci arttext\&pid=S0034-98872014001000011.

38. Johnston FM, Beckman M. Navigating difficult conversations. J Surg Oncol. 2019;120(1):239. DOI: https://doi.org/10.1002/jso.25472.

39. Zwingmann J, Baile WF, Schmier JW, Bernhard J, Keller M. Effects of patient-centered communication on anxiety, negative affect, and trust in the physician in delivering a cancer diagnosis: A randomized, experimental study. Cancer. 2017; 123(16):3167-75. DOI: https://doi.org/10.1002/cncr.30694.

40. Atienza J, Linares M, Padilla M, Morales IM. Breaking bad news to antenatal patients with strategies to lessen the pain: a qualitative study. Reprod Health. 2018; 15(1):11. DOI: https://doi.org/10.1186/s12978-018-0454-2.

41. Toivonen AK, Lindblom-Ylänne S, Louhiala P, Pyörälä E. Medical students' reflections on emotions concerning breaking bad news. Patient Educ Couns. 2017;100(10):1903-9. DOI: https://doi.org/10.1016/j.pec.2 017.05.036.

42. Kübler-Ross E. Sobre la muerte y los moribundos. Barcelona: Grijalbo; 1993.

43. Gao Z. Delivering bad news to patients-the necessary evil. J Med Coll PLA. 2011; 26(2):1038. DOI: https://doi.org/10.1016/S1000-1948(11)60033-6.

44. Maguire P, Pitceathly C. Key communication skills and how to acquire them. Br Med J. 2010; 325:697-700. DOI: https://doi.org/10.1136/bmj.325.7366.697.

45. Wang SY, Chen $\mathrm{CH}$, Chen YS, Huang HL. The attitude toward truth telling of cancer in Taiwan. J Psychosom Res. 2004; 57(1):53-8. DOI: https://doi.org/10.1016/S00223999(03)00566-X.

46. Ishaque S, Saleem T, Khawaja FB, Qidwai W. Breaking bad news: exploring patient's perspective and expectations. J Pak Med Assoc. 2009; 60(5):1-3. Recuperado de https://jpma.org.pk/article-details/2061?article id=2061.

47. Gómez Sancho M. Cómo dar malas noticas en medicina. Ciudad de México: Editorial Manual Moderno; 2019.

48. Benítez MA, Asensio A. Communication with terminally ill patients. Aten Primaria. 2002; 30(7):463-6. DOI: https://doi.org/10.1016/s0212-6567(02)79073-2.

49. Rubiales ÁS, Martín Y, del Valle ML, Garavís MI, Centeno C. Información al enfermo oncológico: Los límites de la verdad tolerable. Cuad Bioética. 1998; 1:45-54. Recuperado de http://aebioetica.org/ revistas/1998/1/33/45.pdf. 
50. Muñoz MT, Sorum PC, Mullet E. Breaking bad news: The patient's viewpoint. Health Commun. 2011; 26(7):649-55. DOI: https://doi.org/10.1080/10410236.2011.561919.

51. Carosella A, Snyder A, Ward E. What parents of children with complex medical conditions want their child's physicians to understand. JAMA Pediatr. 2018; 172(4):315. DOI: https://doi.org/10.1001/jamapediatrics.2017.3931.

52. Paul CL, Clinton-McHarg T, Sanson-Fisher RW, Douglas H, Webb G. Are we there yet? The state of the evidence base for guidelines on breaking bad news to cancer patients. Eur J Cancer. 2009; 45(17):2960-6. DOI: https://doi.org/10.1016/j.ejca.2009.08.013.

53. Gracey C, Gordon G, Haidet P, Helmer D, Harrell H, Blatt B, et al. Not the same everywhere. J Gen Intern Med. 2006; 21(5):405-9. DOI: https://doi.org/10.1111/j.15251497.2006.00417.x.

54. Maguire P, Fairbairn S, Fletcher C. Consultation skills of young doctors: I-Benefits of feedback training in interviewing as students persist. $\mathrm{Br}$ Med J (Clin Res Ed). 1986; 292(6535):1573-6. DOI: https://doi.org/10.1136/bmj.292.6535.1573.

55. Pearl R, Donahue M, Bryan T. The development of tact: Children's strategies for delivering bad news. J Appl Dev Psychol. 1985; 6(2-3):141-9. DOI: https://doi.org/10.1016/01933973(85)90056-5.

56. Rees CE, Sheard CE, McPherson AC. A qualitative study to explore undergraduate medical students' attitudes towards communication skills learning. Med Teach. 2002; 24(3):289-93. DOI: https://doi.org/10.1080/01421590220134123.

57. Vogel D, Meyer M, Harendza S. Verbal and non-verbal communication skills including empathy during history taking of undergraduate medical students. BMC Med Educ. 2018; 18(1):1-7. DOI: https://doi.org/10.1186/s12909-018-1260-9.

58. Borrell F. Entrevista clínica. Manual de estrategias prácticas. Barcelona: SEMFYC ediciones; 2014. Recuperado de Internet, https://www.semfyc.es/wp-content/uploads/2016/05/EntrevistaClinica_Borrell.pdf.

59. Cleland J, Foster K, Moffat M. Undergraduate students' attitudes to communication skills learning differ depending on year of study and gender. Med Teach. 2005; 27(3):246-51. DOI: https://doi.org/10.1080/01421590400029541.

60. Newton JT, Fiske J. Breaking bad news: A guide for dental healthcare professionals. Br Dent J. 1999; 186(6):278-81. DOI: https://doi.org/10.1038/sj.bdj.4800087a.

61. van Osch M, Sep M, van Vliet LM, van Dulmen S, Bensing JM. Reducing patients' anxiety and uncertainty, and improving recall in bad news consultations. Health Psychol. 2014; 33(11):1382-90. DOI: https://doi.org/10.1037/hea0000097

62. Wittenberg-Lyles EM, Goldsmith J, Ragan SL, Sanchez-Reilly S. Medical students' views and ideas about palliative care communication training. Am J Hosp Palliat Care. 2010; 27(1):3849. DOI: https://doi.org/doi:10.1177/1049909109347327.

63. Bain K. Lo que hacen los mejores profesores de universidad. Valencia: Universidad de Valencia; 2007.

64. Brouwers MH, Bor H, Laan R, van Weel C, van Weel-Baumgarten E. Students' experiences with a longitudinal skills training program on breaking bad news: A follow-up study. Patient Educ Couns. 2018; 101(9):1639-44. DOI: https://doi.org/10.1016/j.pec.2018.05.008.

65. Wakefield A, Cocksedge S, Boggis C. Breaking bad news: Qualitative evaluation of an interprofessional learning opportunity. Med Teach. 2006; 28(1):53-8. DOI: https://doi.org/10.1080/01421590500312805.

66. Marteau TM, Humphrey C, Matoon G, Kidd J, Lloyd M, Horder J. Factors influencing the communication skills of first-year clinical medical students. Med Educ. 1991; 25(1):127-34. https://doi/org/10.1111/j.1365-2923.1991.tb00038.x.

67. Barnett M. A GP guide to breaking bad news. Practitioner. 2004; 248(1659):392-403. PMID: 15214269. https://pubmed.ncbi.nlm.nih.gov/15214269/

68. Narayanan V, Bista B, Koshy C. 'BREAKS' protocol for breaking bad news. Indian J Palliat Care. 2010; 16(2):61. DOI: https://doi.org/10.4103/0973-1075.68401. 
69. Baile WF, Buckman R, Lenzi R, Glober G, Beale EA, Kudelka AP. SPIKES - A six-step protocol for delivering bad news: application to the patient with cancer. Oncologist. 2000; 5:302-11. DOI: https://doi.org/10.1634/theoncologist.5-4-302.

70. Campbell EM, Sanson-Fisher RW. Breaking bad news 3: Encouraging the adoption of best practices. Behav Med. 1998; 24(2):73-80. https://doi.org/10.1080/08964289809596383.

71. Garg A, Buckman R. Teaching medical students how to break bad news. Can Med Assoc J. 1997; 156:1159-64. Recuperado de https://www.cmaj.ca/content/cmaj/156/8/1159.full.pdf.

72. Ganesh A, Ganesh G. Reflective writing by final year medical students: Lessons for curricular change. Natl Med J India. 2010; 23(4):226-30. PMID: 21192519. Recuperado de https://www.researchgate.net/publication/

49715729_Reflective_writing_by_final_year_medical_students_Lessons_for_curricular_chang e.

73. Bascuñán $M$, Roizblatt A, Roizblatt D. Comunicación de malas noticias en medicina: un estudio exploratorio. Rev Méd Univ Navarra. 2007 ;51(2):28-31. Recuperado de https://revistas.unav.edu/index.php/revista-de-medicina/article/view/9132/8062.

74. Pang Y, Tang L, Zhang Y, Song L, Goelz T, Fritzsche K, et al. Breaking bad news in China: implementation and comparison of two communication skills training courses in oncology. PsychoOncology. 2014; 611:608-11. DOI: https://doi.org/10.1002/pon.3698.

75. Servotte JC, Bragard I, Szyld D, Van Ngoc P, Scholtes B, Van Cauwenberge I, et al. Efficacy of a short role-play training on breaking bad news in the emergency department. West J Emerg Med. 2019; 20(6):893-902. DOI: https://doi.org/10.5811/westjem.2019.8.43441

76. Guilera T, Batalla I, Jorge S-G. Shadowing patients: experimentar empatía en estudiantes de $\begin{array}{llll}\text { medicina. } & \text { Educ 21(2):112-7. } & \text { DOI: }\end{array}$ https://doi.org/10.1016/j.edumed.2018.06.006.

77. Flores-Funes D, Aguilar-Jiménez J, Lión-Ruiz RJ, Aguayo-Albasini JL. ¿Comunicamos correctamente las malas noticias en medicina? Resultados de un taller de formación basado en videos y debriefing. Educ Médica. 2020;2 1(2):118-22. DOI: https://doi.org/10.1016/j.edumed.2018.07.005.

78. de Moura Villela EF, Bastos LK, de Almeida WSA, Pereira AO, de Paula Rocha MS, de Oliveira FM, et al. Effects on medical students of longitudinal small-group learning about breaking bad news. Perm J. 2020; 24:1-4. DOI: https://doi.org/10.7812/TPP/19.157.

79. Vermylen JH, Wayne DB, Cohen ER, McGaghie WC, Wood GJ. Promoting readiness for residency: Embedding simulation-based mastery learning for breaking bad news into the medicine sub-internship. Acad Med. 2020; 95(7):1050-6. DOI: https://doi.org/10.1097/ACM.0000000000003210.

80. Carrard V, Bourquin C, Stiefel F, Schmid Mast M, Berney A. Undergraduate training in breaking bad news: A continuation study exploring the patient perspective. PsychoOncology. 2020; 29(2):398-405. DOI: https://doi.org/10.1002/pon.5276.

81. Schmitz FM, Schnabel KP, Bauer D, Woermann U, Guttormsen S. Learning how to break bad news from worked examples: Does the presentation format matter when hints are embedded? Results from randomised and blinded field trials. Patient Educ Couns. 2020; (2019):1-6. DOI: https://doi.org/10.1016/j.pec.2020.03.022.

82. Papadakos C, Stringer T, Papadakos J, Croke J, Embleton A, Gillan C, et al. Effectiveness of a multiprofessional, online and simulation-based difficult conversations training program on self-perceived competence of oncology healthcare provider trainees. J Cancer Educ. 2020 Mar 5, doi: https://doi.org/10.1007/s13187-020-01729-x.

83. Kissane DW, Bylund CL, Banerjee SC, Bialer PA, Levin TT, Maloney EK, et al. Communication skills training for oncology professionals. J Clin Oncol. 2012; 30(11):1242-7. DOI: https://doi.org/10.1200/JCO.2011.39.6184.

84. Colletti L, Gruppen L, Barclay M, Stern D. Teaching students to break bad news. Am J Surg. 2001; 182(1):20-3. DOI: https://doi.org/10.1016/S0002-9610(01)00651-1. 
85. Supe A. Interns' perspectives about communicating bad news to patients: a qualitative study. Educ Health (Abingdon). 2011; 24(3):1-6. Recuperado de https://www.researchgate.net/publication/221768409 Interns' Perspectives about_Commun icating Bad News to Patients A Qualitative Study.

86. Karnieli-Miller O, Palombo M, Meitar D. See, reflect, learn more: qualitative analysis of breaking bad news reflective narratives. Med Educ. 2018; 52(5):497-512. DOI: https://doi.org/10.1111/medu.13582.

87. Engerer C, Berberat PO, Dinkel A, Rudolph B, Sattel H, Wuensch A. Specific feedback makes medical students better communicators. BMC Med Educ. 2019; 19(1):1-8. DOI: https://doi.org/10.1186/s12909-019-1470-9.

88. Shapiro J, Kasman D, Shafer A. Words and wards: A model of reflective writing and its uses in medical education. J Med Humanit. 2006; 27(4):231-44. DOI: https://doi.org/10.1007/s10912-006-9020-y.

(C) 2020 por los autores. Enviado para su publicación en acceso abierto bajo los términos y condiciones de la licencia Creative Commons Attribution (CC BY) (http://creativecommons.org/licenses/by/4.0/). 
